\title{
Roles of Ontologies in Cartography
}

\author{
Otakar Čerba* \\ Department of Geomatics, Faculty of Applied Sciences, University of West Bohemia, Plzeň, Czech Republic, cerba@kgm.zcu.cz \\ * Corresponding author
}

Keywords: Ontology, Cartography, Linked Open Data, Semantics

\begin{abstract}
:
Ontologies (in computer science and information science) represent the essential tool for a formalised description of concepts, data, information, knowledge and other entities as well as relations among them. Their history is relatively old. The idea of ontologies in informatics started in the mid-1970s, but ontology as the philosophical discipline connected to existence and nature of reality came from the Ancient Greek. The ontologies as a part of knowledge-based systems were discussed in the 1980s. In 1993 Thomas R. Gruber defined ontology in information science as "a specification of a conceptualisation". After that, the first languages and formats coding ontologies have been developed, and massive construction process of ontologies began. For example, the Basel Register of Thesauri, Ontologies and Classifications presents about 700 ontologies and more the 1000 other tools with a similar character. The theory of ontologies and development as ontologies are entirely on a high level. However, their implementation (especially in several domains) is in its infancy.
\end{abstract}

For example, in the geographical domain, there are many ontologies (called geo-ontologies) such as FAO (Food and Agriculture Organization of the United Nations) Geopolitical Ontology, ontologies of USGS (United States Geological Survey) or ontologies of Ordnance Survey. However, their implementation is usually limited by home organisations, which provide for the management, development and updating of ontologies. In many cases, they are not an integral part of Linked Open Data (LOD). This fact can be considered as the critical shortcoming because only in connection with Linked Open Data and free data sharing and combining the main benefits of ontologies (emphasis on a semantic description, derivation of new knowledge or complete independence) can be fully appreciated.

This document has to describe opportunities for the implementation of ontologies in cartography. The purpose of the implementation of an ontology depends on various types of ontologies. There are defined four essential types of ontologies - upper ontologies, domain ontologies, task ontologies and application ontologies.

Upper and domain ontologies contain general terms (in the case of upper ontologies) and domain-specific terms (in case of domain ontologies). Annotation properties (labels, definitions or comments) usually describe these terms, interconnected by data properties and/or object properties and restricted by logical axioms. Such ontologies are usually provided as vocabularies or thesauri. They can be used in two ways. Domain ontologies can describe cartography as a science or human activity. In previous years several paper and articles were discussing the term "cartography" and its position in Linked Open Data space, including various ontologies, ontological description of cartographic knowledge or ontological comparison of various definitions of the term "map". These activities can aim for the development of a cartographic knowledge base or building of semantic tools such as multilingual thesauri or vocabularies.

The second way consists in the exploitation of domain ontologies containing semantic information about data visualising by a map. In this case, such domain ontology can be used as a tool for development of a legend of a map, especially in a case where a map is focused on particular issues. If such ontology is published as Linked Open Data, it is possible to generate such legend automatically as well as to reflect any changes. Such solution enables an efficient interconnection of cartographers and domain experts. Domain ontologies can be used for a definition of logical rules restricting and describing data, information and knowledge. These rules and knowledge extracted in the reasoning process can be applied during the map development. They can provide information on possible combinations of data or a hierarchy of objects visualising by a map and described by a map legend.

The task ontologies are not focused on a complicated system of classes (representing types of object) as domain ontologies. They are usually based on instances (individuals) representing concrete data objects. Therefore they can be 
used as data resources. However, the overwhelming majority of geo-ontologies does not contain any geometry (coordinates) to enable a visualisation in a map. This apparent disadvantage shows the importance of LOD. If a task ontology is published as 5-star LOD (RDF/Resource Description Framework/ data with interconnection to external data resources published on the Web under an open license), and identity relation (links to equivalent object published in other data sets) are filled, it is possible to find in LOD space geometries as well as other additional information and attributes for visualization.

The remaining type of ontologies is called application ontology. It is a combination of both previous kinds - domain ontology and task ontology. Application ontologies usually provide vocabularies as well as data stored in an ontological structure. Such a combination allows controlling data correctness and integrity by a set of logical rules. This functionality is emphasised by the rich possibilities of the Description Logic (quantifiers or types of relations). Their implementation in cartography corresponds with methods discussed in previous paragraphs. The main advantage of the approach using an application ontology consists in a homogeneous interconnection of data and semantics.

The real implementation of ontologies, other semantic resources and Linked Open Data principles in cartography can make web mapping development process more efficient, because the normalised semantic description enables to automatize many activities, including a derivation of new data and knowledge or checking of data as well as cartographic processes. Such an approach can bring the cartography closer to knowledge bases and systems and realise ideas of real-time cartography.

The research reported in this paper has been supported by the following project - Sustainability support of the centre NTIS - New Technologies for the Information Society, LO1506, Czech Ministry of Education, Youth and Sports. 